\title{
Epigenetic Profiling Provides Insights into AIDS Resistance in
}

\section{African Green Monkey}

\author{
Zheng Yuan, Xiao-jun Zhou
}

Laboratory Animal Center of the Academy of Military Medical Sciences, Beijing, China

Correspondence to: Dr.Xiaojun Zhou . Laboratory Animal Center of the Academy of Military Medical

Sciences, Beijing 100071, China. zhouxiaojun320320@126.com.

Telephone:+86-10-66948217.

Funding: This study was funded by the National Projects of Infectious Disease under Grant

No.2017ZX10304402003 and the National Natural Science Foundation of China under Grant

No.31501908.

Competing Interests: The authors have declared that no competing interests exist.

\section{Abstract}

As a natural host of simian immunodeficiency virus (SIV), African green monkeys (AGM) do not develop AIDS although high levels of SIV replication were maintained. Low frequencies of $\mathrm{CD}^{+} \mathrm{T}$ cells and high frequencies of $\mathrm{CD} 8^{\mathrm{dim}} \mathrm{T}$ cells were observed in healthy adult AGM, which may partially explain the absence of SIV-induced disease progression. Elucidating the mechanisms that allow this natural host co-exist with SIV without progressive disease may facilitate knowledge of AIDS pathogenesis. Here we show: (1) Compared with junior AGM, 3 miRNA were up-regulated in adult AGM in which hsa-miR-151a-3p was 
1 shown to target both $C D 4$ and MAZR; 15 miRNAs were down-regulated in adult AGM in

2 which hsa-miR-140-5p, hsa-miR-126-3p and hsa-miR-194-5p were shown to target $C D 8 \alpha$; (2)

3 MeDIP sequencing analysis of adult AGM samples revealed that hypermethylation exists in

4 the promoter region of $C D 4, C X C R 6, C C R 5$, while hypomethylation exists in the promoter

5 region of $R U N X 3$, ICAM2; (3) Hypomethylation in the promoter region of PTK2 contributes

6 to up-regulated expression of hsa-miR-151a-3p in adult AGM, while hypermethylation in the

7 promoter region of $W W P 2$ contributes to down-regulated expression of hsa-miR-140-5p. Our

8 data for the first time demonstrates the link between miRNA and DNA methylation

9 expression profile, which may together contribute to the phenotype of AIDS resistance in

10 AGM.

\section{Author Summary}

African green monkeys (AGM) do not develop AIDS although high levels of SIV replication

were maintained. Elucidating the mechanisms that allow this natural host co-exist with SIV

without progressive disease may facilitate knowledge of AIDS pathogenesis. In this study, the miRNA expression patterns were found to be associated with the switch from $\mathrm{CD}^{+}$to CD4-CD8 $\mathrm{a}^{\mathrm{dim}}$ in adult AGM. The up-regulated hsa-miR-151a-3p was shown to target both

AGM CD4 and MAZR, while the down-regulated hsa-miR-140-5p, hsa-miR-126-3p and hsa-miR-194-5p were shown to target AGM CD8 $\alpha$. And none of these miRNAs possess target sites in cynomolgus macaque $(\mathrm{CM}) C D 4, C D 8 \alpha$ and $M A Z R$ reflecting differences in AIDS resistance between these two species. Our data also demonstrates the link between miRNA and DNA methylation expression profile, indicating that multiple distinct 
1 mechanisms may contribute to AIDS resistance in AGM. Knowledge of the non-pathogenic

2 nature of SIV infection in AGM may provide insight into development of new therapeutic

3 strategies.

\section{$5 \quad$ Introduction}

6 Simian immunodeficiency viruses (SIV) belong to the group of lentiviruses that infect

7 non-human primates (NHP). Like HIV-1 and HIV-2, all known SIV subtypes use CD4 as a

8 receptor and either CCR5, CXCR4, CCR2 as a co-receptor [1,2,3]. SIV infection of natural

9 hosts, such as AGM, is usually lack of progression to AIDS despite high viraemia, while HIV

10 infection in humans and experimental SIV infection in rhesus macaques (Macaca mulatta)

11 progress to AIDS. One obvious difference between progressive HIV infection and

12 non-progressive SIV infection is the absence of immune activation during the chronic phase

13 of infection in natural hosts [4].

14 Low frequencies of $\mathrm{CD} 4^{+} \mathrm{T}$ cells and high frequencies of $\mathrm{CD} 8^{\mathrm{dim}} \mathrm{T}$ cells have been shown to 15 exist in healthy adult AGM $[5,6]$. CD $8^{\text {dim }} \mathrm{T}$ cells could induce antibody production from $\mathrm{B}$

16 cells in vitro suggesting that $\mathrm{CD} 8^{\mathrm{dim}} \mathrm{T}$ cells might supplement for the lack of $\mathrm{CD} 4^{+} \mathrm{T}$ cells in

17 AGM [7]. Down-regulation of CD4 by memory T cells in adult AGM protects these T cells

18 from infection by SIVagm in vivo [8], but the molecular mechanism remains unclear.

19 Epigenetic phenomena are defined as heritable mechanisms that establish and maintain

20 mitotically stable patterns of gene expression without modifying the base sequence of DNA,

21 which include DNA methylation, post-translational histone modifications and RNA-based

mechanisms including those controlled by small non-coding RNAs (miRNAs) in mammalian 
1 cells [9].

2 This study aims to identify the differential expressed miRNAs, as well as DNA methylation

3 features, between junior and adult AGM, which may provide insights into AIDS resistance in

$4 \quad$ AGM and add knowledge to development of new therapeutic strategies.

6 Materials and Methods

$7 \quad$ Ethics Statement

8 SIVagm-uninfected junior (less than 2 years old) and adult (more than 5 years old) AGM

9 were chosen for analysis. Animals were housed in troop enclosures with an outdoor facility

10 amd fed nonhuman primate chow per day and a combination of fresh bananas, apples, carrots

113 days per week. Toys were available for animals raised for the purpose of environment

12 enrichment. The experiments were conducted in accordance with the recommendations of the

13 ethics provision for experiments on non-human primate of the ethics committee of the

14 Academy of Military Medical Sciences. The protocol was approved by the ethics committee

15 of the Academy of Military Medical Sciences with the identification number 2018032. All

16 protocols were in strict accordance with the recommendations in the Guide for the Care and

17 Use of Laboratory Animals.

\section{PBMC Isolation}

19 Peripheral blood mononuclear cells were isolated from fresh blood by Ficoll gradient 20 separation. $1 \times 10^{7}$ cells were mixed with $600 \mu 1$ mirVana RNA lysis buffer (Ambion, Austin,

21 Texas, USA) to achieve lysis and inactivate endogenous RNAses. Lysates were frozen at -80

${ }^{\circ} \mathrm{C}$ until further processing. 


\section{RNA isolation and Small RNA Deep Sequencing}

2 Total RNA was isolated using the RNeasy mini kit (Qiagen) according to the manufacturers'

3 instructions. Small RNA libraries were created using the TruSeq Small RNA Sample

4 Preparation Kit (Illumina) and sequencing performed using HiSeq 2000 sequencer (Illumina).

5 The small RNA deep sequencing and data analysis were carried out by Shanghai KangChen

6 biotechnology company (Shanghai, China).

\section{Inhibition of endogenous miRNAs}

Locked nucleic acid (LNA)-modified anti-miRs (Exiqon) were used for the inhibition of endogenous miRNAs in PBMCs of junior and adult AGM [10]. PBMC were maintained in RPMI1640 supplemented with $100 \mathrm{U} / \mathrm{ml}$ penicillin, $100 \mu \mathrm{g} / \mathrm{ml}$ streptomycin and $10 \%$ fetal bovine serum (FBS) (Invitrogen). LNA-modified anti-miRs were transfected at a final concentration of $10 \mathrm{nM}$ by Entranster ${ }^{\mathrm{TM}}-\mathrm{R} 4000$ (Engreen Biosystem).

\section{Real-Time Quantitative Reverse-Transcription Polymerase Chain Reaction}

MMLV reverse transcriptase (Takara) was used for the reverse-transcription (RT) reaction and quantitative PCR was performed by an ABI PRISM7500 system (Applied Biosystems).

The RT primers and the primer sets specific for each miRNA amplification are shown in Supplementary Table 1. Expression of selected miRNAs and mRNA of the target genes predicted to be targeted by miRNAs inhibited by anti-miR transfection were measured by qPCR using SYBR green chemistry [11]. Primer sequences for the target genes are listed in Supplementary Table 2 .

\section{DNA isolation and MeDIP sequencing}

DNA was isolated using the QIAamp DNA mini kit (Qiagen) according to the manufacturers' 
1 instructions. Isolated DNA were fragmented to a size range of $\sim 200-500 \mathrm{bp}$ with a Diagenode

2 Bioruptor. About $1 \mu \mathrm{g}$ of fragmented DNA was prepared for Illumina HiSeq 4000 sequencing

3 as the following steps: 1) End repair of DNA samples; 2) A single 'A' base was added to the

$4 \quad 3$ ' ends; 3) Illumina's genomic adapters were ligated to DNA fragments; 4) DNA fragments

5 were immunoprecipitated by anti-5-methylcytosine antibody; 5) Immunoprecipitated DNA

6 fragments were amplified by PCR amplification; 6) Size selection of 300-600bp DNA

7 fragments using AMPure XP beads. The completed libraries were quantified by Agilent 2100

8 Bioanalyzer. The libraries were denatured with $0.1 \mathrm{M} \mathrm{NaOH}$ to generate single-stranded

9 DNA molecules, captured on Illumina flow cell, amplified in situ. The libraries were then

10 sequenced on the Illumina HiSeq 4000 following the HiSeq 3000/4000 SBS Kit (300 cycles)

11 protocol.After sequencing images generated, the stages of image analysis and base calling were performed using Off-Line Basecaller software (OLB V1.8). After passing Solexa

CHASTITY quality filter, the clean reads were aligned to Chlorocebus-sabaeus genome

(Applied Biosystems). Primer sequences for $C D 4, R U N X 3$ and $A C T B$ were listed in 
1 Supplementary table 3. Quantitative PCR was performed in a $25 \mu$ reaction volume with $12.5 \mu 1$ of $2 \times$ SYBR Green PCR Master Mix (Applied Biosystems), 2.5 pmol of each primer, and $50 \mathrm{ng}$ of bisulfite-treated DNA sample. Thermal cycling was as follows: $95^{\circ} \mathrm{C}$ for $10 \mathrm{~min}$, 40 cycles of $95^{\circ} \mathrm{C}$ for $15 \mathrm{~s}, 60^{\circ} \mathrm{C}$ for $1 \mathrm{~min}$. The amount of methylated DNA (percentage of methylated reference) was calculated as follows: ratio of quantity of target gene to quantity of target gene of test sample divided quantity of $A C T B$.

\section{$7 \quad$ Statistical Analysis}

Values were expressed as mean $\pm \mathrm{SD}$. The comparative CT method was used in real-time qRT-PCR assay according to the delta-delta CT method. Statistical analyses were performed using GraphPad Prism version 5.01. t test was used to compared statistical differences. The data were considered statistically significant at $P<0.05$.

\section{Results}

\section{Differential miRNA expression in PBMCs from junior and adult AGM}

Different from junior AGM, low frequencies of $\mathrm{CD}^{+} \mathrm{T}$ cells and high frequencies of $\mathrm{CD} 8^{\mathrm{dim}}$ $\mathrm{T}$ cells were observed in healthy adult AGM, which may partially explain the absence of SIV-induced disease progression. To investigate the role of miRNA in this process, we profiled the PBMC miRNA expression in 3 junior AGM and 3 adult AGM. As shown in Figure 1a, 3 miRNAs were up-regulated while 15 miRNAs were down-regulated with over 1.5-fold change in adult AGM $(P<0.05)$. To validate the profiling data, miRNAs in Table 1 were further analyzed via quantitative RT-PCR between PBMCs from 5 junior AGM and 5 adult AGM. Results showed a 3.6-fold decrease for hsa-miR-215-5p $(P<0.01)$, a 2.0 2.5-fold 
1 decrease for hsa-miR-194-5p, hsa-miR-99b-5p, hsa-miR-125a-5p (all $P<0.01$ ), a 2.0 2.5-fold

2 increase for hsa-miR-95-3p $(P<0.01)$, a 1.5 2.0-fold decrease for hsa-miR-126-3p,

3 hsa-miR-181b-5p, hsa-miR-140-5p, hsa-miR-199a-3p, hsa-miR-10a-5p (all $P<0.01$ ), and a

$4 \quad$ 1.5 2.0-fold increase for hsa-miR-151a-3p, hsa-miR-10b-5p (all $P<0.05)$ (Figure 1b and 1c).

\section{Effects of selected miRNAs on AGM CD4, CD8 $\alpha$ and $M A Z R$}

6 As MAZR is a protein suppressor of the $C D 8 \alpha$ enhancer region [12], we investigate whether

7 the above miRNAs target AGM CD4,CD8 $\alpha$ and MAZR. hsa-miR-151a-3p was predicted to target AGM CD4 and MAZR while hsa-miR-140-5p, hsa-miR-126-3p and hsa-miR-194-5p were predicted to target AGM CD8 (Figure 2a).Then, the potential miRNA-target pairs were examined by inhibiting these endogenous miRNAs in PBMCs using LNA-modified anti-miRs. Anti-miR treatment may cause the increase on the abundance of the target mRNA if it is indeed suppressed by the endogenous miRNA via mRNA degradation. As shown in Figure $2 \mathrm{~b}$, the selected miRNAs were all validated to target AGM CD4, CD8 $\alpha$ and MAZR, respectively. Interestingly, none of these miRNAs possess target sites in cynomolgus macaque (CM) $C D 4, C D 8 \alpha$ and $M A Z R$, which may partially explain the differences in AIDS resistance between these two species.

\section{Differential DNA methylation in PBMCs from junior and adult AGM}

Besides miRNA profiles, we wondered whether differences also exist in DNA methylation profiles and performed MeDIP sequencing. Genes with significantly different DNA methylation signals in promoter regions were selected and the Top10 biological processes (BP) were listes through Gene Ontology (GO) analysis (Figure 3). RUNX3 is required for the establishment of epigenetic silencing of CD4 in cytotoxic-lineage thymocytes [13].Our 
1 results showed hypomethylation in $R U N X 3$ promoter and up-regulation of its mRNA in adult

2 AGM (Figure 4b and 4f), as well as hypermethylation in CD4 promoter (Figure 4a), which together may account for the CD4 repression in adult AGM T cells. Although CD8 $\alpha$ was over-expressed in adult AGM T cells, we did not find similar DNA methylation changes in proteins regulating CD8 expression, such as MAZR, TOX, Ikaros [14], suggesting other mechanisms may exist. As a coreceptor used by SIV in vivo, the level of CCR5 is very low on CD4+ $\mathrm{T}$ cells of natural host species $[15,16]$. CXCR6 is another potential alternative coreceptor for SIV as an efficient entry pathway in vitro [17,18]. Analysis through QSG-MSP demonstrated both hypermethylation in CXCR6 and CCR5 promoters (Figure 4c and 4d) and both mRNAs were down-regulated in adult AGM PBMCs (Figure 4f), providing another insight into AIDS resistance in African green monkey. Recently, the genome of another natural host, sooty mangabeys (Cercocebus atys), has been sequenced and assembled [19]. Also, the C.atys immune-regulatory protein intercellular adhesion molecule 2 (ICAM-2) was found to possess a major structural change in exons 3-4 and expression of this variant leads to reduced cell surface expression of ICAM-2. Our results showed that hypomethylation in promoter region of ICAM-2 existed in adult AGM (Figure 4e) and expression of ICAM-2 was significantly up-regulated in adult AGM (Figure 4f). Furthermore, hypomethylation in the promoter region of $P T K 2$, as well as hypermethylation in the promoter region of $W W P 2$ hsa-miR-140-5p was located, respectively. This indicates a cross-link between roles of DNA methylation and miRNA expression in AIDS resistance in AGM. 


\section{Discussion}

2 Natural hosts have co-evolved with SIV and are capable of avoiding disease progression, of

3 which mechanisms may diverge. Unlike AGM, sooty mangabey (SM) maintains healthy

4 frequencied of CD4+ $\mathrm{T}$ cells and genome sequencing has identified two gene products

5 (ICAM-2 and TLR-4), which show structural differences that may influence cell-surface

6 expression (ICAM-2) and downstream signalling (TLR-4) [19]. In AGM, the co-evolution

7 with SIVagm may be accounted for partially by the development of CD4-CD8 $\mathrm{a}^{\mathrm{dim}} \mathrm{T}$ cells

8 from memory $\mathrm{CD}^{+} \mathrm{T}$ cells. Like other natural hosts of SIV, CD4-like immunological

9 functions can be elicited by CD4- $\mathrm{T}$ cells in $\mathrm{AGM}[20]$ and preservation of $\mathrm{CD}^{+} \mathrm{T}$ cell

10 function may contribute to the lack of immune activation in AGM and SM [5,21,22].

11 It has been shown that memory $\mathrm{CD}^{+} \mathrm{T}$ cells down-regulate CD4 and up-regulate CD8 $\alpha$ [8],

12 but the actual mechanism(s) underlying the switch from $\mathrm{CD}^{+}$to $\mathrm{CD} 4-\mathrm{CD} 8 \mathrm{a}^{\mathrm{dim}}$ remains

unclear. Previous studies have demonstrated genetic differences between certain regulatory elements from AGM compared to other primates [23]. miRNAs result in translational suppression of suppression of target mRNA in all known animal and plant genomes [24]. DNA methylation is an epigenetic modification typically associated with stable transcriptional silencing and plays an important role in several biological processes associated with development and disease $[25,26]$. In this study, the miRNA expression

19 patterns were found to be associated with the switch from $\mathrm{CD}^{+}$to $\mathrm{CD} 4 \mathrm{CD} 8 \mathrm{a}^{\mathrm{dim}}$ in adult AGM. The up-regulated hsa-miR-151a-3p was shown to target both AGM CD4 and MAZR, while the down-regulated hsa-miR-140-5p, hsa-miR-126-3p and hsa-miR-194-5p were 
macaque $(\mathrm{CM}) C D 4, C D 8 \alpha$ and $M A Z R$ reflecting differences in AIDS resistance between these two species. Differential DNA methylation in promoter regions of $C D 4, R U N X 3$, CXCR6, CCR 5, ICAM-2, as well as PTK2 and WWP2, was also demonstrated indicating that multiple distinct mechanisms may contribute to AIDS resistance in AGM. Knowledge of the non-pathogenic nature of SIV infection in AGM may provide insight into development of new therapeutic strategies.

\section{ABBREVIATIONS}

AIDS, acquired immune deficiency syndrome; MAZR, Myc-associated Zn finger-related factor; MeDIP, methylation DNA immunoprecipitation; CXCR6, C-X-C motif chemokine receptor 6; CCR5, C-C motif chemokine receptor 5; RUNX3, Runt-related transcription factor 3; ICAM2, intercellular cell adhesion molecule-2; CCR2, C-C motif chemokine receptor 2; MMLV, moloney murine leukemia virus; ACTB, actin $\beta$; TLR-4, Toll-like receptor 4; PTK2, protein tyrosine kinase 2; WWP2, ww domain-containing protein 2 .

\section{Acknowledgments}

The authors are grateful to Professor Jun-feng Li (Institute of Microbiology and Epidemiology, Beijing, China) for technical support in QSG-MSP.

\section{Author Contributions}

Conceived and designed the experiments: XZ ZY. Performed the experiments: XZ JW. Wrote the paper: XZ.

\section{References}

1. Ling B, Veazey RS, Marx PA (2008). Nonpathogenic CCR2-tropic SIVrcm after serial passage and its effect on SIVmac infection of Indian rhesus macaques. Virology 379:38-44.

2. Zhang Y, Lou B, Lal RB, Gettie A, Marx PA, et al.(2000) Use of inhibitors to evaluate coreceptor usage by simian and simian/human immunodeficiency viruses and human immunodeficiency virus type 2 in primary cells. J Virol 74:6893-6910.

3. Beer BE, Foley BT, Kuiken CL, Tooze Z, Goeken RM, et al.(2001) Characterization of novel simian immunodeficiency viruses from red-capped mangabeys from Nireria (SIVrcmNG409 and -NG411). J Virol 75:12014-12027.

4. Pandrea I, Apetrei C, Dufour J, Dillon N, Barbercheck J, et al.(2006) Simian imunodeficiency virus SIVagm. sab infection of Caribbean African green monkeys: a new model for the study of SIV pathogenesis in natural hosts. J Virol 80:4858-4867. 
5. Pandrea IV, Gautam R, Ribeiro RM, Brenchley JM, Butler IF, et al.(2007)Acute Loss of Intestinal CD4+ T-cells Is Not Predictive of Simian Immunodeficiency Virus Virulence. J Immunol 179:3035-3046.

6. Murayama Y, Amano A, Mukai R, Shibata S, Matsunaga S, et al.(1997) CD4 and CD8 expressions in African green monkey helper T lymphocytes: implication for resistance to SIV infection. Int Immunol 9:843-851.

7. Murayama Y, Mukai R, Inoue-Murayama M, Yoshikawa Y (1999) An African green monkey lacking peripheral CD4 lymphocytes that retains helper T-cell activity and coexists with SIVagm. Clin Exp Immunol 117:504-512.

8. Beaumier CM, Harris LD, Goldstein S, Klatt NR, Whitted S, et al.(2009) Down-regulation of CD4 by memory $\mathrm{CD}^{+} \mathrm{T}$ cells in vivo renders African green monkeys resistant to progressive SIVagm infection. Nat Med 15(8):879-885.

9. Udali S, Guarini P, Moruzzi S, Choi SW, Friso S (2013) Cardiovascular epigenetics: From DNA methylation to microRNAs. Mol Aspects Med 34(4):883-901.

10. Mladinov D, Liu Y, Mattson DL, Liang M (2013) MicroRNAs contribute to the maintenance of cell-type-specific physiological characteristics:miR-192 targets Na+/K+-ATPase $\beta 1$. Nucleic Acids Res 41:1273-1283.

11. Kriegel AJ, Fang Y, Liu Y, Tian Z, MIadinov D, et al.(2010) MicroRNA-target pairs in human renal epithelial cells treated with transforming growth factor beta1: a novel role of miR-382. Nucleic Acids Res 38(22):8338-8347.

12. Bilic I, Koesters C, Unger B, Sekimata M, Hertweck A, et al.(2006) Negative regulation of CD8 expression via $\mathrm{Cd} 8$ enhancer-mediated recruitment of the zinc finger protein MAZR. Nat Immunol 7:392-400.

13. Taniuchi I, Osato M, Egawa T, Sunshine MJ, Bae SC, et al. (2002) Differential requirements for Runx proteins in CD4 repression and epigenetic silencing during T-lymphocyte development. Cell 111:621-634. 14. Kioussis D, Ellmeier W (2002) Chromatin and CD4, CD8A and CD8B gene expression during thymic differentiation. Nat Rev Immunol 2(12):909-919.

15. Paiardini M, Cervasi B, Reyes-Aviles E, Micci L, Ortiz AM, et al.(2011) Low levels of SIV infection in sooty mangabey central memory $\mathrm{CD}^{4+} \mathrm{T}$ cells are associated with limited CCR5 expression. Nat Med 17(7):830-836.

16. Cartwright EK, McGary CS, Cervasi B, Micci L, Lawson B, et al. Divergent CD4+ T memory stem cell dynamics in pathogenic and nonpathogenic simian immunodeficiency virus infections. J Immunol 192:4666-4673.

17. Wetzel KS, Yi Y, Elliott ST, Romero D, Jacquelin B, et al.(2017) CXCR6-mediated simian immunodeficiency virus SIVagmSab entry into Sabaeus African green monkey lymphocytes implicates widespread use of non-CCR5 pathways in natural host infections. J Virol 91(4).pii:e01626-16.

18. Riddick NE, Wu F, Matsuda K, Whitted S, Ourmanov I, et al.(2015) Simian immunodeficiency virus SIVagm efficiently utilizes non-CCR5 entry pathways in African green monkey lymphocytes: potential role for GPR15 and CXCR6 as viral coreceptors. J Virol 90(5):2316-2331.

19. Palesch D, Bosinger SE, Tharp GK, Vanderford TH, Paiardini M, et al.(2018) Sooty mangabey genome sequence provides insight into AIDS resistance in a natural SIV host. Nature 553(7686):77-81.

20. Vinton C, Klatt NR, Harris LD, Briant JA, Sanders-Beer BE, et al.(2011) CD4-like immunological function by CD4- $\mathrm{T}$ cells in multiple natural hosts of simian immunodeficiency virus. J Virol 85(17):8702-8708.

21. Gordon SN, Klatt NR, Bosinger SE, Brenchley JM, Milush JM, et al.(2007) Severe Depletion of 
Mucosal CD4+ T-cells in AIDS-Free Simian Immunodeficiency Virus-Infected Sooty Mangabeys. J Immunol 179:3026-3034.

22. Brenchley JM, Paiardini M, Knox KS, Asher AI, Cervasi B, et al.(2008) Differential Th17 CD4 T-cell depletion in pathogenic and nonpathogenic lentiviral infections. Blood 112:2826-2835.

23. Matsunaga S, Mukai R, Inoue-Murayama M, Yoshikawa Y, Murayama Y (2000) Sequence and functional properties of African green monkey CD4 silencer. Immunol Lett 75:47-53.

24. Carthew RW, Sontheimer EJ (2009) Origins and Mechanisms of miRNAs and siRNAs. Cell 136(4):642-655.

25. Siegfried Z, Eden S, Mendelsohn M, Feng X, Tsuberi BZ,et al.(1999) DNA methylation represses transcription in vivo. Nat Genet 22:203-206.

26. Cedar H, Bergman Y ( 2009 ) Linking DNA methylation and histone modification: patterns and paradigms. Nat Rev Genet 10:295-304.

\section{Supporting Information Legends}

\section{Figure 1. miRNA profiling and validation of junior and adult AGM PBMCs.}

(A) Heat map of miRNA microarray expression data from PBMC samples of junior AGM $(\mathrm{n}=3)$ and adult AGM $(\mathrm{n}=3)$. $(\mathbf{B} \& \mathbf{C})$ Validation of miRNA microarray data by quantitative reverse-transcription polymerase chain reaction. The relative expression of miRNAs was normalized to expression of the internal control (U6). The $P$ values were calculated by 2-sided Student $t$ test. ${ }^{*} P<0.05$; ${ }^{* *} P<0.01$.

Figure 2. Summary of miRNA-target pairs supported by the anti-miR transfection

experiment. The mRNA abundance for the predicted targets was significantly increased in PBMC after inhibition of the indicated endogenous miRNA. The $P$ values were calculated by 2-sided Student $t$ test. ${ }^{*} P<0.05$.

Figure 3. Enrichment map of GO categories for biological processes. A indicates those hypermethylated genes in adult AGM while B indicates those hypomethylated genes. Colors 
1 represent $P$ values on a $\log$ scale (with red corresponding to the most highly significant,

$2 \quad P<0.05)$. Node size represents the number of genes in a category.

4 Figure 4. Comparative analysis of differential DNA methylation between junior and

adult AGM. (A,B,C,D\&E) Quantitative analysis of the levels of CpG DNA methylation of

CD4, RUNX3, CXCR6, CCR5 and ICAM2. (f) Quantitative RT-PCR analysis for mRNA stands for $P<0.05$.

Figure 5. Quantitative analysis of the levels of CpG DNA methylation of PTK2 and

WWP2. (A\&B) Quantitative analysis of the levels of CpG DNA methylation of PTK2 and

WWP2. (C) Schematic show of the cross-link between DNA methylation and miRNA

12 expression.

Table 1. Properties of miRNAs differentially expressed in PBMCs of adult and junior African green monkeys.

Supplementary Table 1. Sequences of the primers used in the SYBR-green-based quantitative RT-PCR validation.

Supplementary table 2. Primer Sequences for quantitative analysis of AGM CD4,CD8a, 21 MAZR. 
A

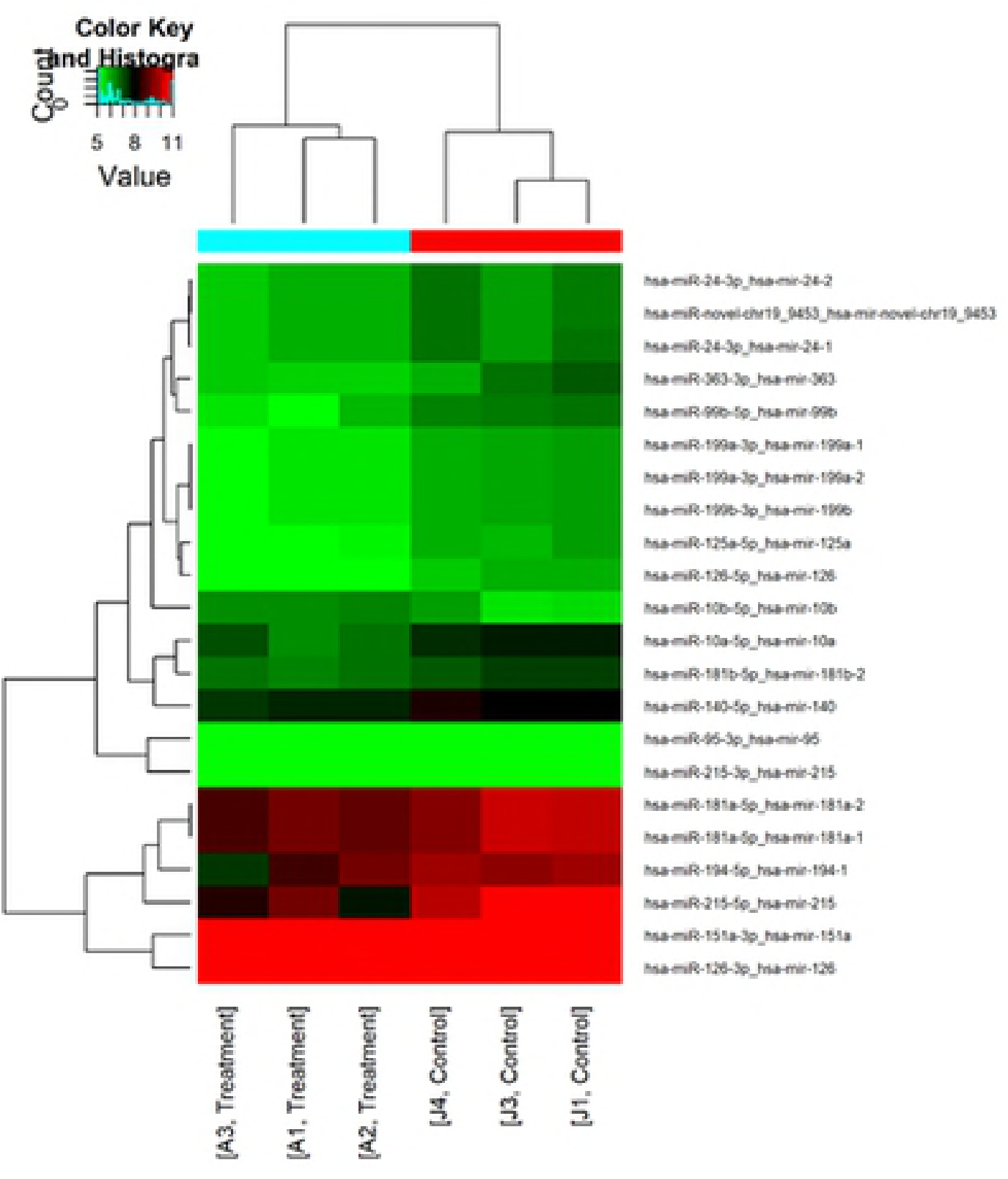

B
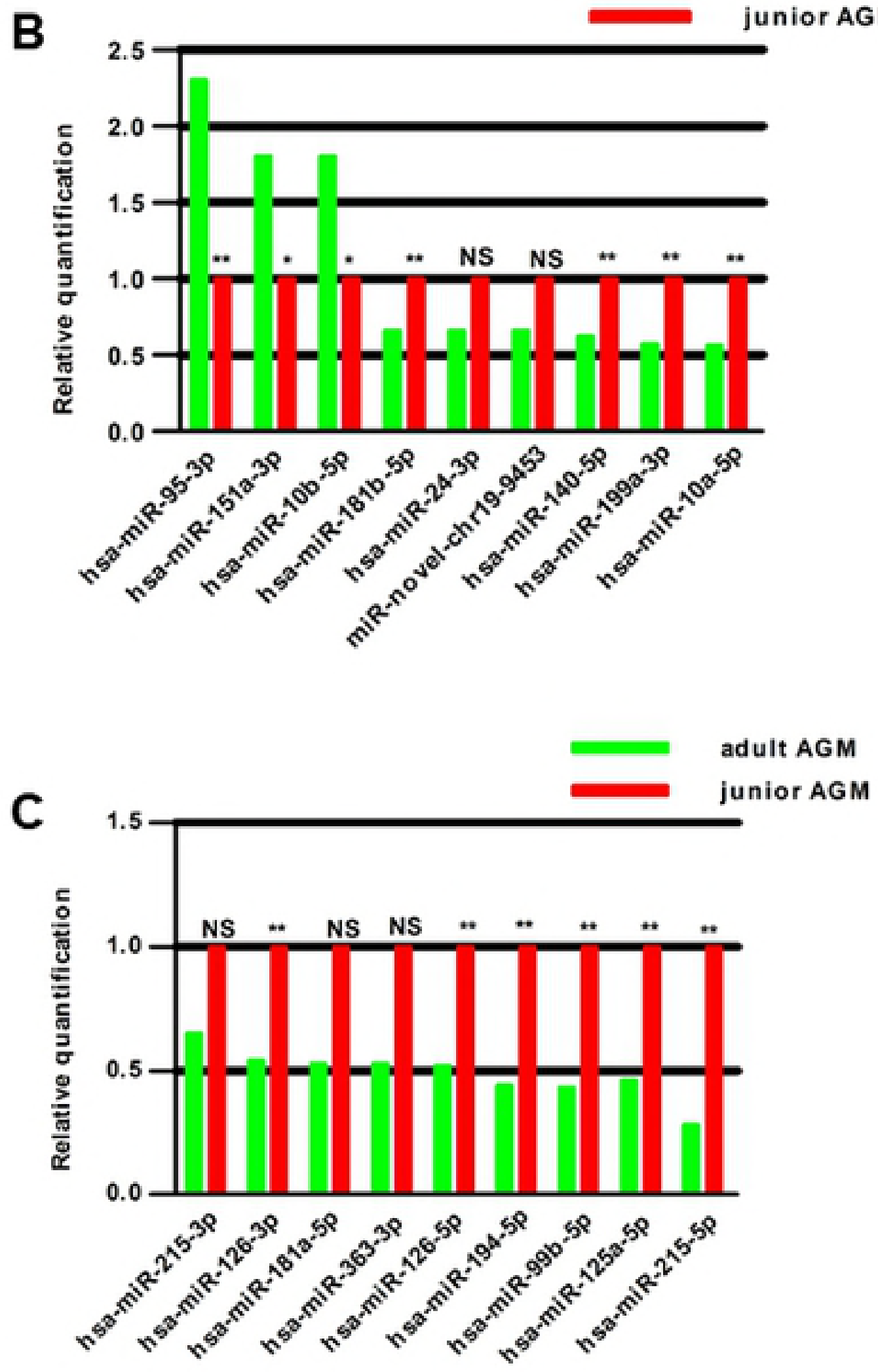
B
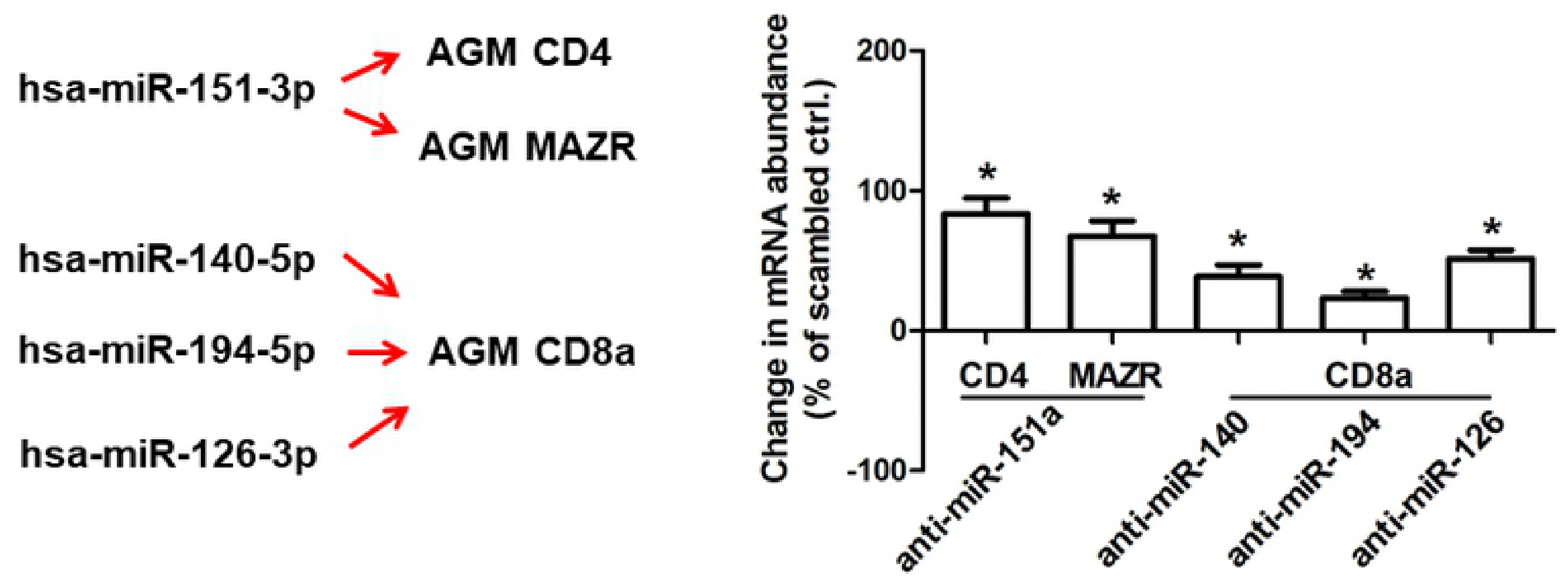
A

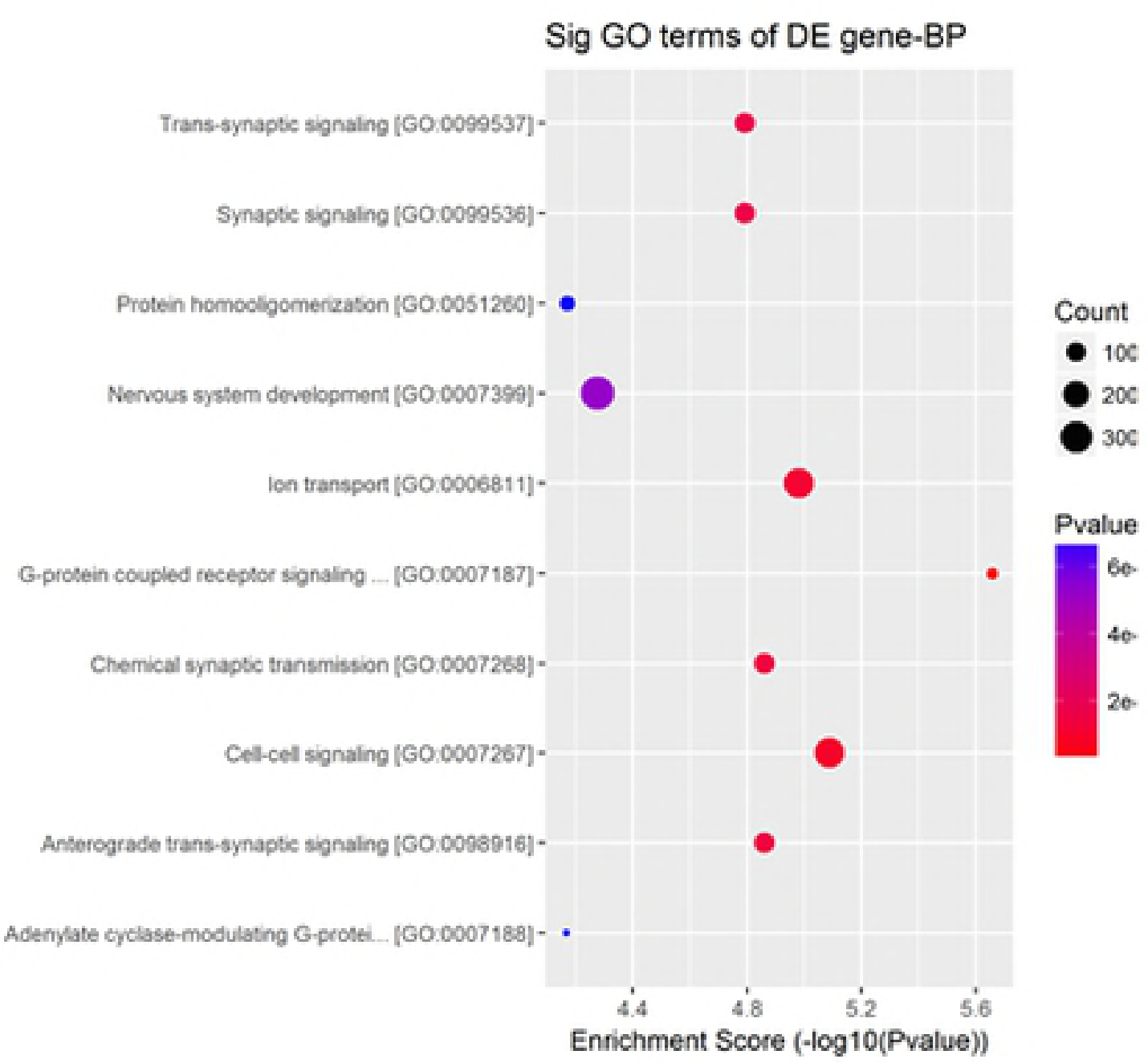

B

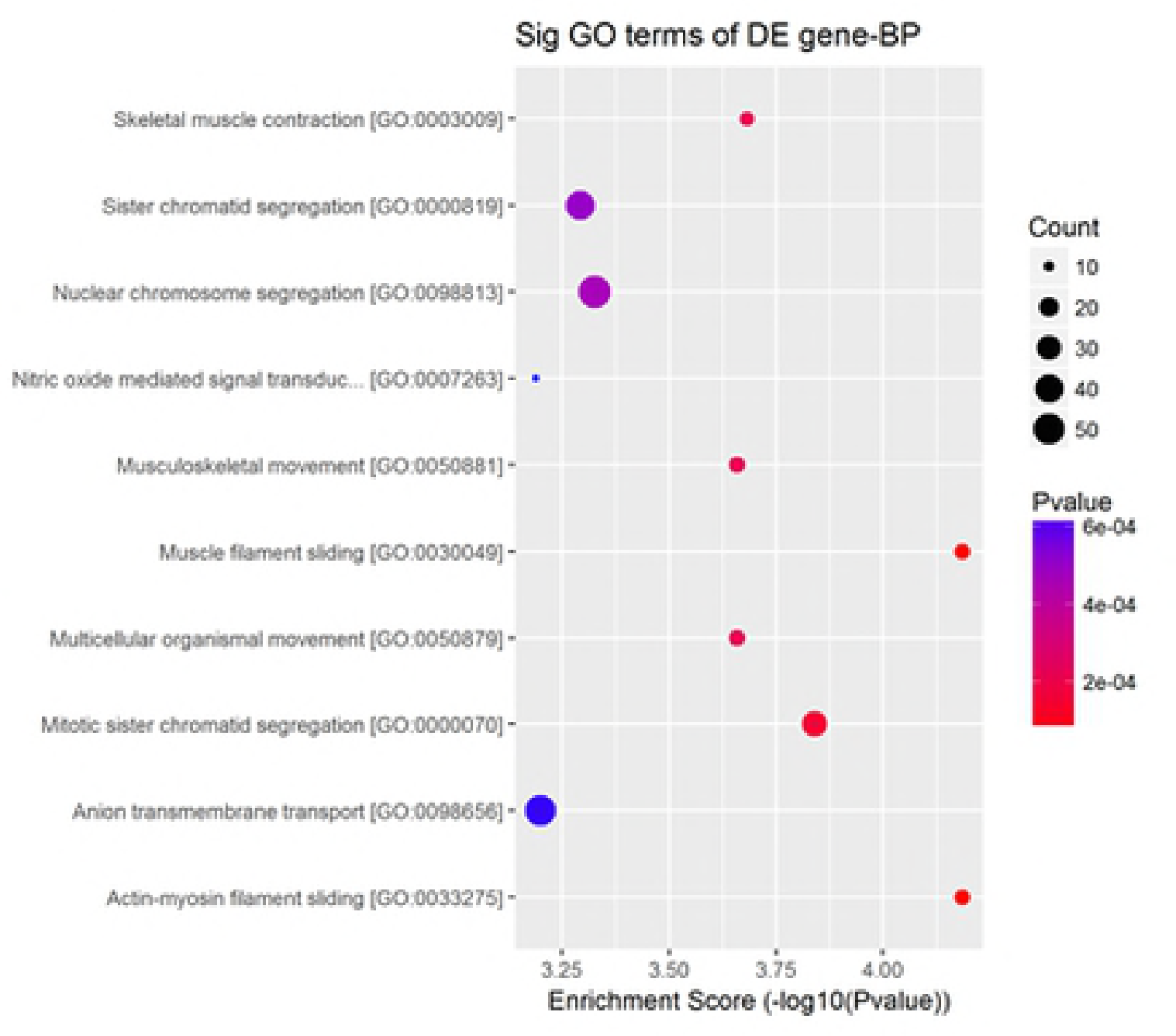


A

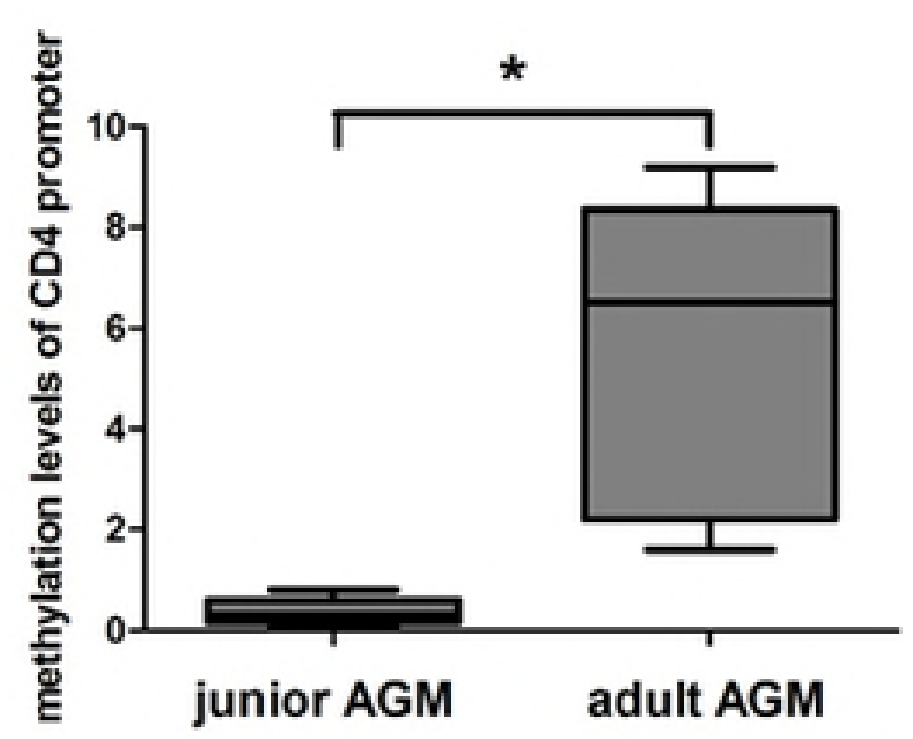

D

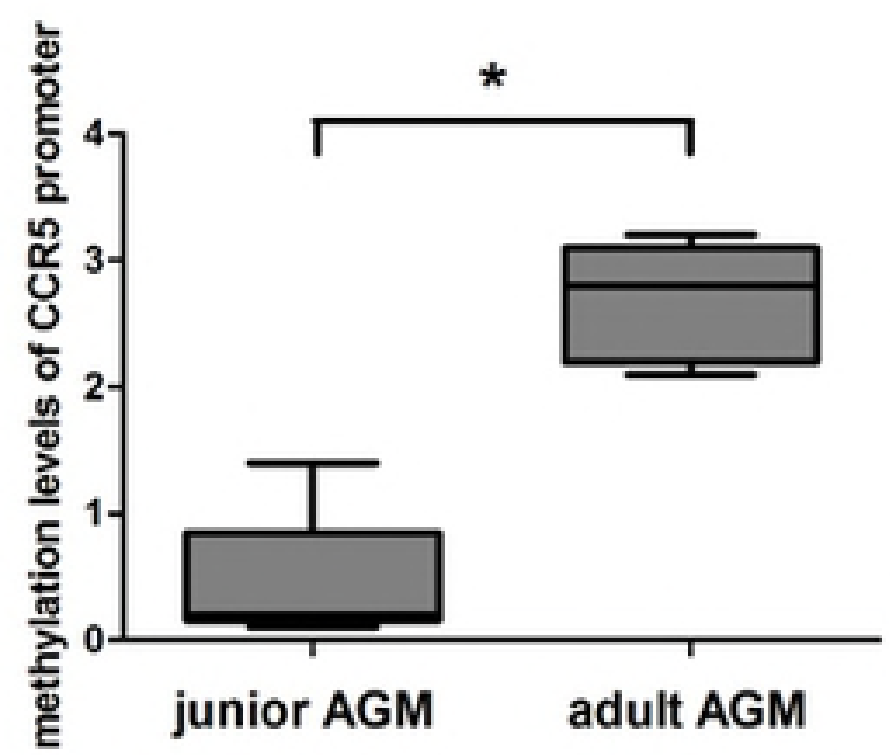

B

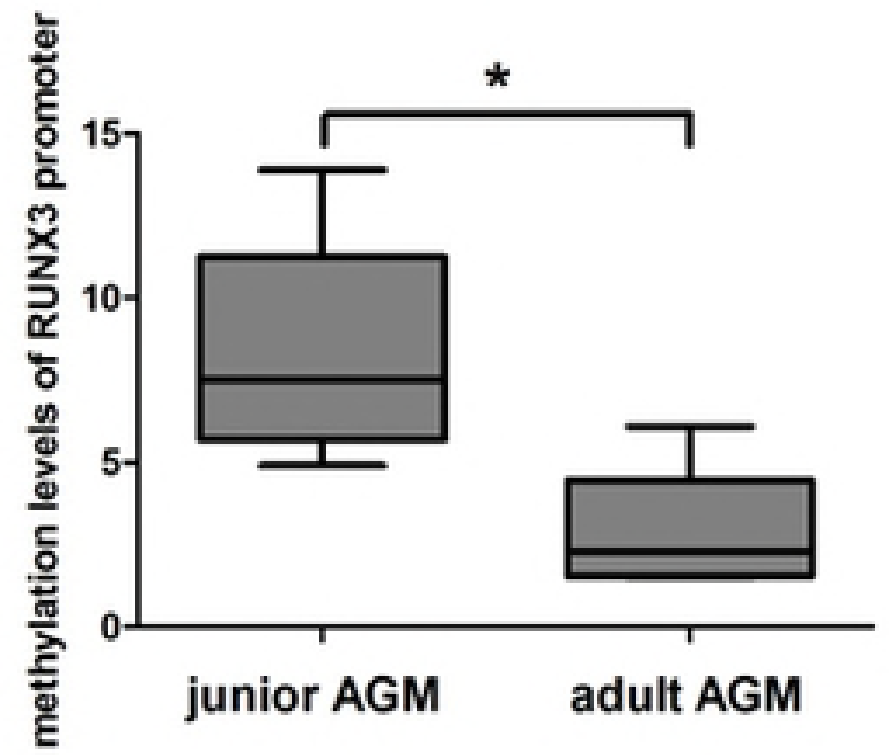

E

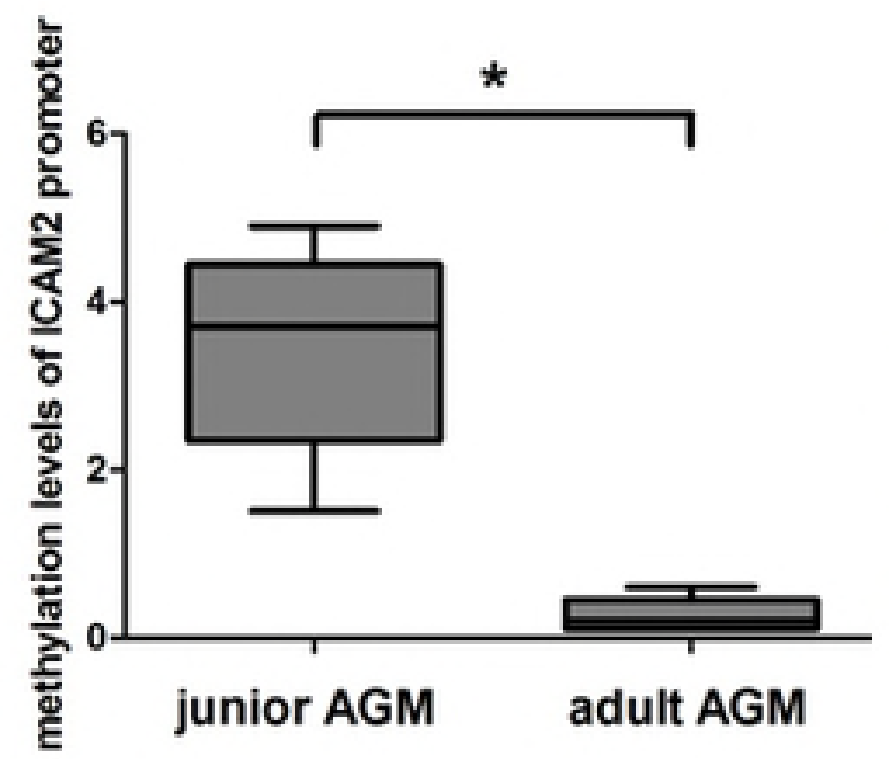

C

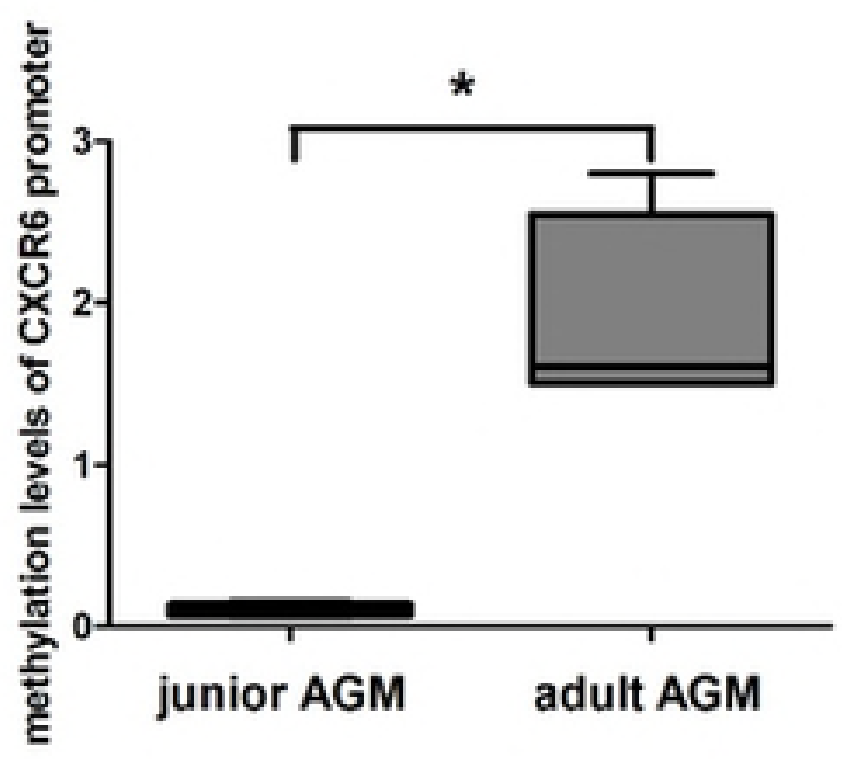

F

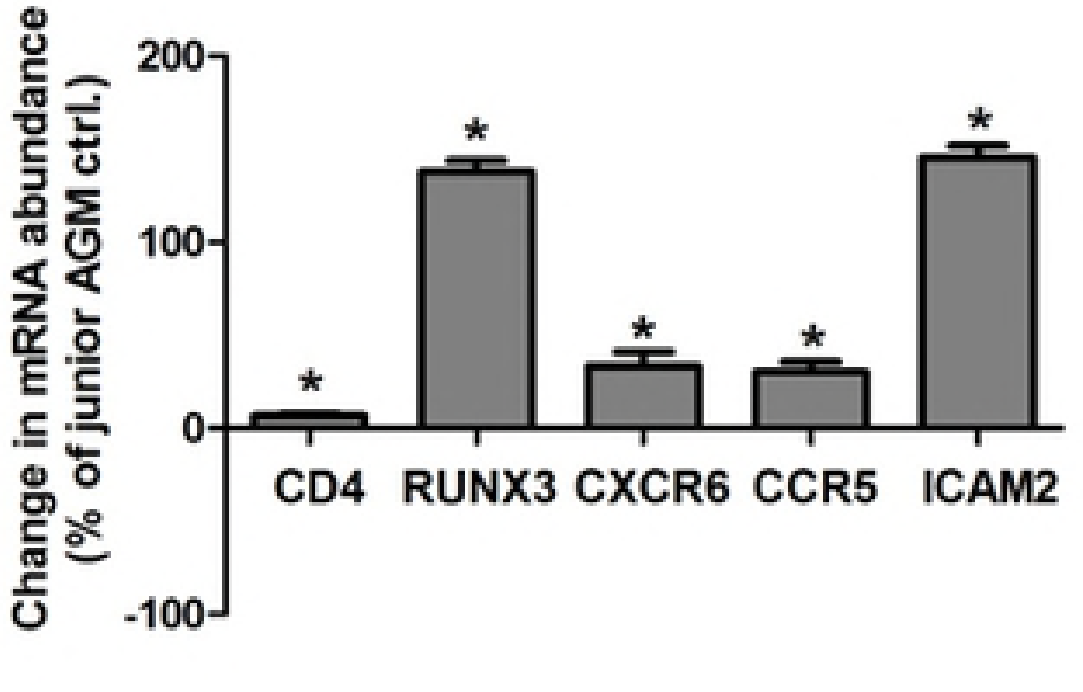


A

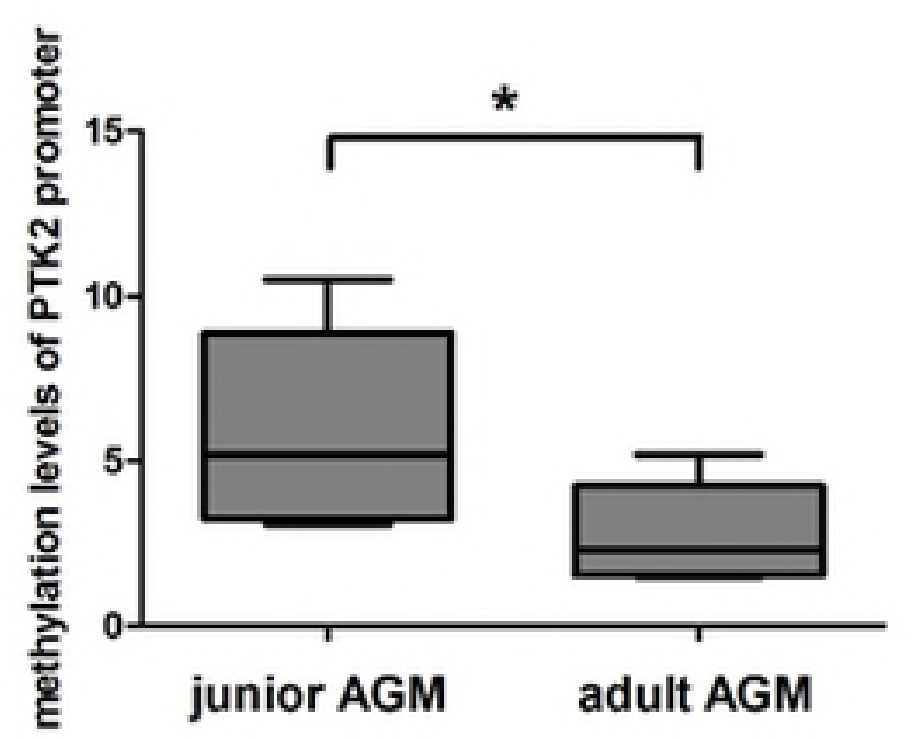

B

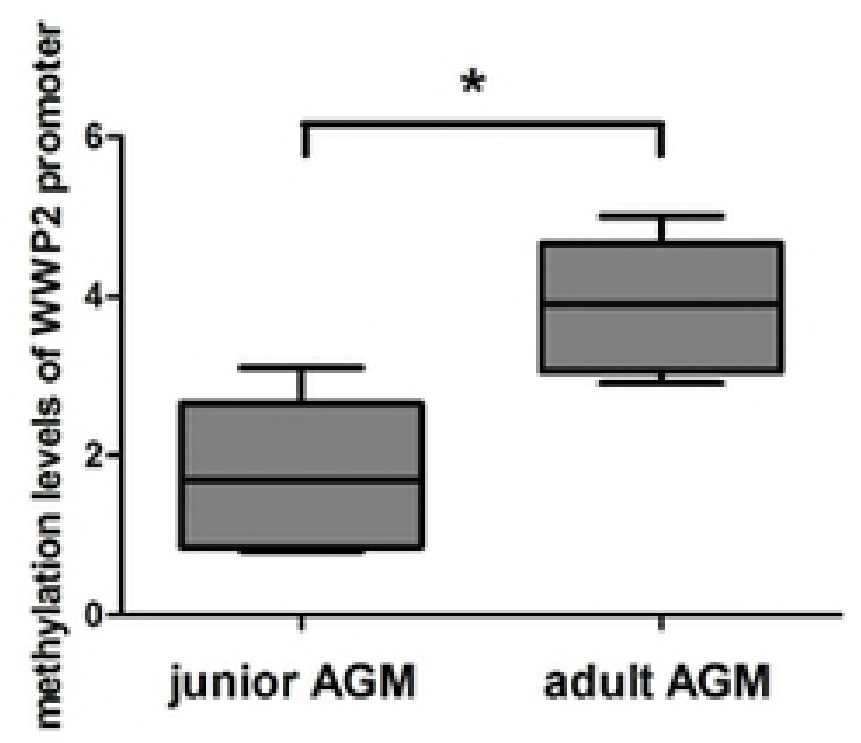

C

PTK2 $\rightarrow$ hsa-miR-151-3p

WWP2 $\rightarrow$ hsa-miR-140-5p 